\title{
Gravitational Waves and Dark Energy
}

\author{
Peter L. Biermann ${ }^{1,2,3,4,5}$, Benjamin C. Harms ${ }^{1}$ \\ ${ }^{1}$ Department of Physics and Astronomy, The University of Alabama, Box 870324, Tuscaloosa, AL 35487-0324, USA \\ ${ }^{2}$ MPI for Radioastronomy, Bonn, Germany \\ ${ }^{3}$ Karlsruhe Institute of Technology (KIT) - Institut für Kernphysik, Germany \\ ${ }^{4}$ Department of Physics, University of Alabama at Huntsville, AL, USA \\ ${ }^{5}$ Department of Physics $\&$ Astronomy, University of Bonn, Germany
}

Corresponding author: bharms@bama.ua.edu

\begin{abstract}
The idea that dark energy is gravitational waves may explain its strength and its time-evolution. A possible concept is that dark energy is the ensemble of coherent bursts (solitons) of gravitational waves originally produced when the first generation of super-massive black holes was formed. These solitons get their initial energy as well as keep up their energy density throughout the evolution of the universe by stimulating emission from a background, a process which we model by working out this energy transfer in a Boltzmann equation approach. New Planck data suggest that dark energy has increased in strength over cosmic time, supporting the concept here. The transit of these gravitational wave solitons may be detectable. Key tests include pulsar timing, clock jitter and the radio background.
\end{abstract}

Keywords: cosmology - dark energy - black holes - gravitational waves.

\section{Introduction}

Dark energy was originally detected as accelerated expansion seen in the distance scale for supernovae of type Ia (Schmidt et al., 1998, Riess et al. 1999, Perlmutter et al. 1999; for a review see Frieman et al. 2008). Many suggestions have been made about what dark energy is, what its strength is, what its time evolution is, and what possible further observational results are.

The idea that dark energy is gravitational waves may explain its strength and its time-evolution. One possible concept is that dark energy is the ensemble of coherent bursts (solitons) of gravitational waves originally produced when the first generation of supermassive back holes was formed (Caramete \& Biermann 2010); the energy density of such solitons would suffice within the uncertainties. These solitons get their initial energy as well as keep up their energy density throughout the evolution of the universe by stimulating emission from a background (Biermann \& Harms 2013). Our model of the background metric resembles the Randall-Sundrum ideas (1999a, b) but is timedependent, and describes the energy flow from the background (strong-gravity) brane to our world (weakgravity) brane. Planck data suggest that dark energy has increased in strength over cosmic time (Planck 2013 XVI), as predicted by our model. Gravitational waves were far below today's dark energy at the epoch of early nucleosynthesis and of the formation of the microwave background ripples (as summarized in Ligo+Virgo-Coll. 2009), both much earlier than the likely formation epoch of the first generation of super-massive black holes. Our model is also consistent with early star formation (Biermann et al. 2014), as we argue below. The transit of the gravitational wave solitons postulated here may be detectable. We discuss the predictions briefly below and elsewhere. We focus on the Boltzmann equation approach, working out the energy transfer from the strong gravity background in stimulated emission.

\subsection{Gravitational solitons from black hole mergers}

Inspired by Bekenstein's (1973) considerations we posit: When the first generation of super-massive black holes was formed, each produced a coherent burst of solitonlike gravitational waves which combine to give a total energy of order

$$
\sim \frac{1}{2} N_{B H, 0} M_{B H} c^{2}\left(1+z_{\star}\right)^{3} .
$$

In the following we also call this an ensemble of soliton waves, or shell fronts. $N_{B H, 0}$ is the original comoving density of super-massive black holes. Today supermassive black holes have a density of $10^{-1.7 \pm 0.4} \mathrm{Mpc}^{-3}$ 
Gravitational Waves and Dark Energy

above $M_{B H}=3 \cdot 10^{6} \mathrm{M}_{\odot}$ (Caramete \& Biermann 2010); assuming that they grow by merging, and allowing for statistical and systematic errors, an original comoving density of $N_{B H, 0}=1 \mathrm{Mpc}^{-3}$ seems possible. This comoving density is the density black holes had at the beginning, so transposed to today without change in their numbers per comoving volume. The data suggest that there was a generation of first super-massive black holes with a mass between $M_{B H} \sim 10^{6} M_{\odot}$ and $M_{B H} \sim 10^{7} M_{\odot}$. The original black hole mass may be $\sim 3 \cdot 10^{6} M_{\odot}$ considering (i) the black hole mass function (Greene et al. 2006, Caramete \& Biermann 2010), (ii) the instability of massive stars (Appenzeller \& Fricke 1972a, b) in an agglomeration picture (Spitzer 1969, Sanders 1970), and (iii) the observed black hole in our Galactic Center (e.g. Eckart et al. 2005). The redshift of creation $z_{\star}$ may be large, as formation of massive stars may begin at redshift 80 (Biermann \& Kusenko 2006). Redshifts $z_{\star}$ from about 30 to 50 allow a quantitative interpretation of the data of dark energy. At the original density of black holes adopted here redshift 50 is consistent with the mass of $M_{B H} \sim 3 \cdot 10^{6} M_{\odot}$, and redshift 30 would imply $M_{B H} \sim 10^{7} M_{\odot}$, in either case to make the estimate consistent with dark energy today.

What is the motivation for considering gravitational waves? Bekenstein (1973) wrote about the entropy of the universe: “... we must regard black hole entropy as a genuine contribution to the entropy content of the universe". However, entropy is also information, and information must have a carrier. A natural suggestion is that this carrier is gravitational waves, with an energy commensurate with the black hole scale. This suggestion is consistent with the fact that cosmological black holes are not in thermodynamic equilibrium, and therefore the entropy associated with such black holes should be described by statistical mechanics as advocated in Harms and Leblanc $(1992,1993)$. This speculation immediately gives

$$
\frac{S}{k_{B}}=N_{G W, 0}=4 \pi\left(\frac{M_{B H}}{m_{P l}}\right)^{2}, \text { for zero spin, }
$$

where $N_{G W, 0}$ is the number of gravitons at the formation of the black hole. For $M_{B H}=3 \cdot 10^{6} \mathrm{M}_{\odot}$ this is $N_{G W, 0} \simeq 10^{90} . M_{B H}$ is the original mass of the black hole, $m_{P l}$ is the Planck mass, $c$ is the speed of light, and $G_{N}$ is Newton's constant of gravity. $E_{G W}$ is the average graviton energy given by

$$
E_{G W}=\frac{1}{8 \pi} \frac{\hbar c^{3}}{G_{N} M_{B H}}=\frac{c^{2}}{8 \pi} \frac{m_{P l}^{2}}{M_{B H}} .
$$

This gives a graviton energy of $E_{G W} \simeq 10^{-30} \mathrm{erg}$ for this black hole mass. The entire energy content then is

$$
N_{G W, 0} E_{G W}=\frac{1}{2} M_{B H} c^{2} .
$$

We picture this as a coherent burst of gravitational waves, or a soliton wave, ejected at formation of the black hole. It is clear from the considerations above that we are not using the weak-field approximation. Multiplying with the original density of super-massive black holes reproduces our estimate above.

\section{$1.2 \quad$ Five-dimensional background model}

In our model for the background, which has some similarity to the Randall-Sundrum (1999a, b) ideas, we identify a possible local metric to describe a $5 \mathrm{D}$ world with a $4 \mathrm{D}$ strong gravity brane and our 4D world weakgravity brane.

$$
\begin{aligned}
d s^{2} & =-e^{(u / l)^{m} t / \psi} c^{2} d t^{2}+\mathrm{e}^{\left(\frac{u}{l}\right)^{p} t / \beta} d u^{2} \\
& +\mathrm{e}^{\left(1-b\left(\frac{u}{l}\right)^{n}\right) 2 t / \alpha t_{H}} \mathrm{e}^{-\left(\frac{u}{l}\right)^{k}\left(1-\frac{t}{\phi}\right)} d x_{i} d x^{i},
\end{aligned}
$$

where $i=1,2,3, \tau_{H}$ is the Hubble time, $l=l_{P l}$ is the Planck length, $\tau_{P l}=l_{P l} / c$ is the Planck time, $u$ is the coordinate in the fifth dimension, and the remaining, non-coordinate quantities are arbitrary parameters. Although the five-dimensional covariant divergence of the energy-momentum tensor does not vanish everywhere, it vanishes on the weak-gravity (weak) brane $(u=0)$, and it is approximately zero on the strong-gravity (strong) brane $(u=l)$ for very small dimensionless ratios $\beta / \tau_{H}$ and $\psi / \tau_{H}$ with $\psi<0$ and $\beta>0$, with the conditions a) $\tau_{H}(\psi+\beta) /(\psi \beta)>>1$, b) $2(b-1) / \alpha>\tau_{H} / \phi-1$, and c) $\alpha^{2}=3$. $g_{00}$ in the metric above then defines the confining potential for the strong brane. Gravitons from our brane stimulate transitions between bound states on the strong brane, resulting in the emission of gravitons onto our brane. This metric describes a weak brane for our world which is expanding with time, and a strong brane which is contracting with time, albeit very slowly for the latter brane. The $5 \mathrm{D}$-cosmological constant measured on the weak brane is $\Omega_{w e a k}=-\left(\frac{\tau_{P l}}{\tau_{H}}\right)^{2}$.

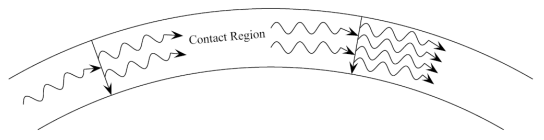

Figure 1: The stimulated emission of gravitons in a shell

In our model the gravitons on the strong-gravity brane obey a Planck-like distribution with Planck temperature. We adopt the point of view that the Planck scales are limits: nothing can go below Planck time 
and Planck length, and no single particle can go beyond Planck energy, in any frame. The strong brane is stable against collapse, since given a Planck spectrum for any wavelength $\lambda$ the free-fall time scale $\tau_{f f}$ is always either equal or longer than the pressure wave time scale $\tau_{s}$.

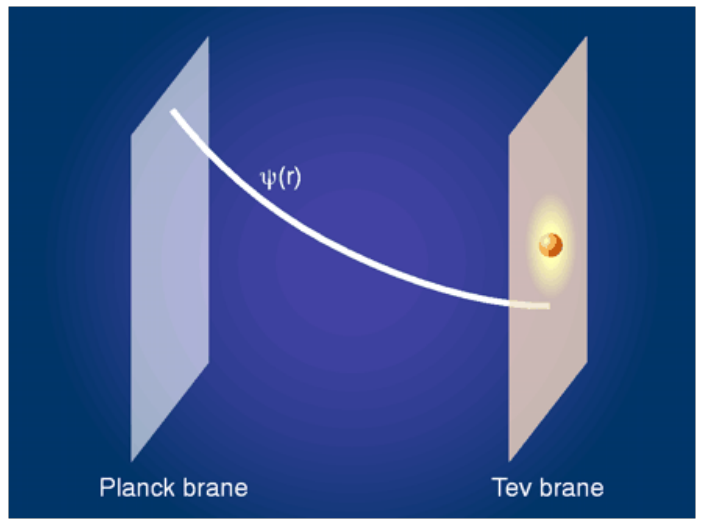

Figure 2: The strong-gravity (Planck brane) and weak-gravity (Tev brane) branes

$$
\tau_{f f}=\tau_{P l}\left(\frac{\lambda}{l_{P l}}\right)^{3 / 2} \geq \tau_{P l}\left(\frac{\lambda}{l_{P l}}\right)=\tau_{s} .
$$

\section{Stimulate Emission of Energy from the Strong-Gravity Brane}

In the following we use a particle-wave duality for gravitons at high energy, which thus associates a wavenumber $\vec{k} / \hbar$ and a corresponding length-scale $\lambda$ to each spatial direction, and we assume localization is possible to about a wavelength.

\subsection{Rate of energy transfer}

The distribution function $\mathcal{N}(k, t)$ is the distribution of occupied allowed states on our brane for gravitons with momenta $k=|\vec{k}|$ and $p=|\vec{p}|$ at time $t$ on the shell and satisfies the equation

$$
\begin{gathered}
\left(\frac{\partial}{\partial t}-\frac{\dot{R}(t)}{R(t)} k \frac{\partial}{\partial k}\right) \mathcal{N}(k, t)= \\
\frac{1}{k} \int \frac{d^{3} k^{\prime}}{(2 \pi)^{3} 2 k^{\prime}} \int \frac{c d^{3} p}{(2 \pi)^{3} 2 E(p)} \int \frac{c d^{3} p^{\prime}}{(2 \pi)^{3} 2 E\left(p^{\prime}\right)} \int \frac{d^{3} k^{\prime \prime}}{(2 \pi)^{3} 2 k^{\prime \prime}} \\
\gamma^{2}|M|^{2}(2 \pi)^{4} \delta^{4}\left(K+Q-K^{\prime}-K^{\prime \prime}-Q^{\prime}\right) \\
(2 \pi)^{6} \delta^{3}\left(\overrightarrow{k^{\prime}}-\overrightarrow{k^{\prime \prime}}\right) \delta^{3}\left(\vec{k}-\overrightarrow{k^{\prime}}\right) \\
{\left[g_{b}\left(p^{\prime}, t\right)(1+\mathcal{N}(k, t))\left(\left(\mathcal{N}\left(k^{\prime}, t\right)+1\right)\left(\mathcal{N}\left(k^{\prime \prime}, t\right)+1\right)-1\right)\right.} \\
\left.-\mathcal{N}(k, t) g_{b}(p, t)\left(1+\mathcal{N}\left(k^{\prime}, t\right)\right)\left(1+\mathcal{N}\left(k^{\prime \prime}, t\right)\right)\right]
\end{gathered}
$$

where $K=(k, \vec{k}), \gamma=k_{r e f, 1}^{3}$, and $Q=(E, \vec{p}) . \quad k_{r e f, 1}$ is a reference momentum to be determined below. The $\delta$-functions, $\delta^{3}\left(\overrightarrow{k^{\prime}}-\overrightarrow{k^{\prime \prime}}\right)$ and $\delta^{3}\left(\vec{k}-\overrightarrow{k^{\prime}}\right)$, have been inserted to impose coherence of the outgoing gravitons. $|M|^{2}$ is the matrix element squared for the quadrupole emission of a graviton of 4-momentum $k^{\prime \prime}$, and has the dimensions of (momentum $)^{-3}$ (time $)^{-1}$. We will assume that this matrix element squared is proportional to $k^{5} \cdot g_{b}(p, t)$ is the occupation number distribution of the background particle sea. $R(t)=\left(1+z_{\star}\right) /(1+z)$ is the scale factor for an expanding universe. The following analysis is done in the observer frame.

The Boltzmann equation for $\mathcal{N}(k, t)$ to lowest order in the expansion of the 4 -dimensional $\delta$-function is

$$
\begin{aligned}
\left(\frac{\partial}{\partial t}-\frac{\dot{R}(t)}{R(t)} k \frac{\partial}{\partial k}\right) & \mathcal{N}(k, t) \simeq \\
& +\frac{+\kappa}{k} \mathcal{N}(k, t)(\mathcal{N}(k, t)+1),
\end{aligned}
$$

where the factor $\kappa$ is given, after integration over all the $\delta$-functions, by

$$
\kappa=\frac{k_{r e f, 2}^{2} H(z)}{2^{4} \pi k_{B H}}|\bar{M}|^{2} \ln \left\{\frac{k_{B H+}}{k}\right\} .
$$

In the equation above $k_{B H+}$ is the maximum momentum at which stimulated emission of gravitons occurs, just above the momentum of the peak of $\mathcal{N}(k, t)$. Since the log-term in eq. 8 varies very slowly over the range of $k$ of interest we approximate this term with a constant and set $\beta=\left\{\ln \left(k_{B H+} / k\right)\right\} /\left(2^{4} \pi\right)$. $|M|^{2}$ is related to $|\bar{M}|^{2}$ by extracting the factors $\left(k / k_{r e f, 1}\right)^{3} / k_{r e f, 1}^{3}, H(z)$. A threshold function of $2 g_{b} \mathcal{N} /\left(g_{b}+\mathcal{N}\right)$ arises from the normalized interaction between the gravitons on the background brane and our brane; this function connects to the strong brane only if $\mathcal{N}>g_{b}$, which is the condition for stimulated emission. These choices do not introduce new constraints or additional assumptions.

Next we redefine $\left(k / k_{r e f, 2}\right)^{2} \bar{\kappa}=\kappa$ to extract the $k$-dependence from $|\bar{M}|^{2}$.

Making the change of variables $k=\tilde{k} / R(t)$, eq.7 can be written as

$$
\frac{\partial \mathcal{N}(\tilde{k}, t)}{\partial t} \simeq \frac{H(z) \tilde{k} \beta}{k_{B H} R(t)} \mathcal{N}(\tilde{k}, t)(\mathcal{N}(\tilde{k}, t)+1) .
$$

In terms of the frequency of the wave at emission this equation is

$$
\frac{\partial \mathcal{N}\left(\nu_{0}, t\right)}{\partial t} \simeq \frac{H(z) h \nu_{0} \beta}{k_{B H} R(t) c} \mathcal{N}\left(\nu_{0}, t\right)\left(\mathcal{N}\left(\nu_{0}, t\right)+1\right) .
$$

Introducing the dimensionless variables

$$
x=\frac{h \nu_{0}}{k_{B} T_{g 0}}, \quad \text { and } \quad y=\int_{0}^{t} \frac{k_{B} T_{g 0} H(z) \beta}{k_{B H} c R(t)} d t^{\prime},
$$

where $k_{B H} c=k_{B} T_{g 0}=m_{P l} c^{2} \frac{m_{P l}}{8 \pi M_{B H}}$. Eq.(10) becomes

$$
\frac{\partial \mathcal{N}}{\partial y} \simeq+x \mathcal{N}(x, y)(\mathcal{N}(x, y)+1)
$$

The solution of this equation is

$$
\mathcal{N}(x, y)=\frac{1}{e^{x(a-y)+b}-1},
$$


Gravitational Waves and Dark Energy

where $a$ and $b$ are constants, to be determined later. This distribution (eq.13) is Planck-like with a timedependent normalized temperature $1 / a$. The rate at which energy is created can be calculated from the expression for $\mathcal{N}$ in eq.10.

The rate at which energy is created can be calculated from the expression for $\mathcal{N}$ in eq.(10). The rate of energy creation per existing graviton (of the total number $\left.N_{G W, 0} R(t)^{4}\right)$ is

$$
\begin{aligned}
& \frac{d<E>}{d t}=\frac{\beta H(z)}{R(t)} \times \\
& \int x^{3} h \nu_{0} \mathcal{N}\left(\nu_{0}, t\right)\left(\mathcal{N}\left(\nu_{0}, t\right)+1\right) d x .
\end{aligned}
$$

The total rate of energy creation is

$$
\frac{d<E_{T}>}{d t}=N_{G W, 0} R(t)^{3} k_{B H} c H(z) \beta A,
$$

where

$$
A=\int x^{4} \mathcal{N}(x, t)(\mathcal{N}(x, t)+1) d x
$$

Inserting all these constants into the integral for $y$ demonstrates that $y$ approaches a constant for the redshift $z_{\star}$ being large, and integrating down to today or even into the future, when $y$ approaches a constant of $\beta<<1$. $a$ is equivalent to an inverse temperature, and should be of order unity. Without loss of generality we can set $b=0$. This integral strongly depends on the exact value of $a-y$, and is of order 30 for $a-y \simeq 1$, and $b$ approaching zero.

The matrix element $|\bar{M}|$ does not evolve with time, and scales as momentum

$$
|\bar{M}|=\epsilon_{M}\left(\frac{k}{m_{P l} c}\right) .
$$

Above we have used $\epsilon_{M}=1$; we now generalize and allow $\epsilon_{M}$ to be different from unity. Writing $|\bar{M}|$ in this way suggests that the interaction between the gravitons on our brane and the gravitons on the background brane comes down to a fundamental coupling constant. This behavior is consistent with the idea [22], that the gravitational coupling strongly increases with energy to approach the other three coupling constants at near Planck energies.

This allows the expression for $\frac{\left.d<E_{T}\right\rangle}{d t}$ to be consistent with the observed energy density under the condition that $A \beta \epsilon_{M}=3$. For the constant $a=1$ above, $\beta$ of order 0.1 , and $\epsilon_{M}=1$, the quantity $\left\{A \beta \epsilon_{M}\right\}$ is in fact close to 3 . However, if we were to require that the $k$-range be very large, then $\beta$ would be larger, and $\epsilon_{M}$ would be required to be smaller than unity accordingly.

Inserting this parameter dependence into eq.(15) then leads back, to within the approximation that
$A \beta \epsilon_{M}=3$, to the result we were seeking,

$$
\frac{3}{2} M_{B H} c^{2} H(z)\left(\frac{1+z_{\star}}{1+z}\right)^{3} .
$$

After integrating we obtain with this redshift dependence a constant dark energy density as in eq.( 1) by multiplying by the redshift evolution of black holes $N_{B H, 0}(1+z)^{3}$. The factor of 4 multiplying $\rho_{D E}$ in eq. 22 below derives from the sum of dark energy density and pressure, and corresponds to $3\left(\rho_{D E}+P_{D E} / c^{2}\right)$. Therefore the rate of change of dark energy with time is $3\left(\rho_{D E}+P_{D E} / c^{2}\right) H(t)$ and today

$$
3 \rho_{D E} H(z=0)=\frac{3}{2} M_{B H} c^{2} H(z=0)\left(1+z_{\star}\right)^{3} .
$$

This justifies the $\rho_{D E} H(t)$ term in eqs.22 and 25. This shows that dark energy remains at the level of eq.(1) throughout the evolution of the universe, in the approximation that most early super-massive black holes were formed over a short span of time.

\subsection{Equation of state}

We define $\rho_{D E}(t, u)$ as the dark energy density, $\rho(t, u)$ as the total energy density, $P(t, u)$ as the total pressure, and we use the equation of state $P_{D E}(t, u)=$ $\rho_{D E}(t, u) c^{2} / 3$. The dark energy density $\rho(t, u)$ is scaled to the value of the dark energy density observed on our brane today ( redshift $z=0$ ).

The Friedmann-Robertson-Walker form of the Einstein equations on the weak-gravity brane must be

$$
(H(t))^{2}=\left(\frac{R(t)}{R(t)}\right)^{2}=\frac{8 \pi G_{N}}{3} \rho(t, 0)
$$

and

$$
\begin{aligned}
\frac{\ddot{R}(t)}{R(t)}= & -\frac{4 \pi G_{N}}{3}\left(\rho(t, 0)+3 \frac{P(t, 0)}{c^{2}}\right) \\
& +\frac{16 \pi G_{N}}{3} \rho_{D E}(t, 0)+\frac{4 \pi G_{N} S_{i n j}}{3 H(t)}
\end{aligned}
$$

where the rate of change of the energy density used is

$$
\begin{aligned}
\rho(t, 0)= & -3\left(\rho(t, 0)+\frac{P(t, 0)}{c^{2}}\right) H(t) \\
& +4 \rho_{D E}(t, 0) H(t)+S_{i n j} .
\end{aligned}
$$

We emphasize that in the second equation, eq.21, the term $16 \pi G_{N} \rho_{D E}(t, 0) / 3$ corresponds to the continuous energy transfer by stimulated emission on the basis of existing dark energy; the additional term $\left(4 \pi G_{N} S_{i n j}\right) /(3 H(t))$ describes new formation of dark energy. This allows a different equation of state for exactly the same cosmological observations, as now for $P_{D E}=\rho_{D E} c^{2} / 3$ the modified equation 21 becomes identical to the canonical version of this equation for 
$P_{D E}=-\rho_{D E} c^{2}$. The corresponding set of equations for the strong-brane are

$$
H_{S B}(t)^{2}=\frac{8 \pi G_{N}}{3}\left(\rho_{D E}\left(t, l_{S B}\right)-\Lambda_{S B}\right)
$$

where $\Lambda_{S B}$ is the cosmological constant on the strong brane at the beginning of the epoch of black hole formation and

$$
\begin{aligned}
& \frac{\ddot{R}_{S B}(t)}{R_{S B}(t)}=-\frac{16 \pi G_{N}}{3} \rho_{D E}(t, 0) \frac{H(t)}{H_{S B}(t)} \\
+ & \frac{8 \pi G_{N}}{3}\left(3 \rho_{D E}\left(t, l_{S B}\right)-\Lambda_{S B}\right)-\frac{4 \pi G_{N} S_{i n j}}{3 H_{S B}(t)}
\end{aligned}
$$

The rate of change of the energy density on the strongbrane is correspondingly

$$
\begin{aligned}
\dot{\rho}_{D E}\left(t, l_{S B}\right)= & 3\left(\rho_{D E}\left(t, l_{S B}\right)+\frac{1}{c^{2}} P_{D E}\left(t, l_{S B}\right)\right) H_{S B}(t) \\
& -4 \rho_{D E}(t, 0) H(t)-S_{i n j} .
\end{aligned}
$$

Eq.24 is derived from eq.23 using the conservation of energy-momentum equations; eqs. 22 and 25 insure that energy is conserved between the two branes, the 4-dimensional boundaries of the 5-dimensional universe described by our model. For epochs before the energy transfer started $\rho_{D E}(t, 0)=0$, and $S_{i n j}=0$, and we can set $\rho_{D E}\left(t, l_{S B}\right)=\Lambda_{S B}$ for $t<t\left(z=z_{\star}\right)$.

\section{Comparison to Experimental Limits}

Our model can be tested by several different types of experiment. We discuss two of these below.

\subsection{Pulsar timing experiments}

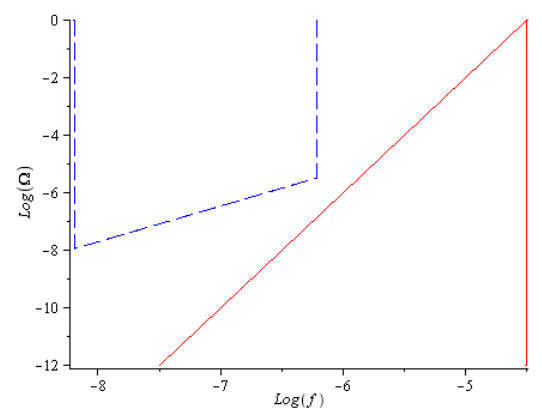

Figure 3: Gravitational wave background limit from pulsar timing (dashed line), and our inferred gravitational wave background from stimulated emission of gravitational waves from the background Planck sea constituting dark energy (straight line). The ordinate is the fraction of closure density $\Omega$ per log bin of frequency, and the abscissa is the frequency of the gravitational waves $f$.
The gravitational waves in our model arise from the production of black holes in the early universe at a redshift of $z \simeq 50$. The observed constant dark energy density is maintained by the continuous production of gravitational waves by black-hole interactions with the Planck sea background. For an idealized model in which all black holes were created at the same time, and with the same mass, the gravitational wave background peaks near $f_{G W, \max } \simeq 10^{-4.5} \mathrm{~Hz} M_{B H, 6.5}^{-1}(1+50) /(1+$ $\left.z_{\star}\right)$.

A soliton comes past a given point in space-time on the order of every 20 seconds. Wave forms are created by uncorrelated solitons passing by a given point. Ultra-precise timing experiments which test the steadiness of timing over time scales of order a few seconds to a few minutes would show these variations in the energy density if the precision is high enough. The precision to detect such a signal has to correspond to seconds in the expansion rate of the universe, which requires the precision to be of order $10^{-17.5}$, or a few $10^{-18}$. This precision is expected to be reached in the next generation of clocks [16].

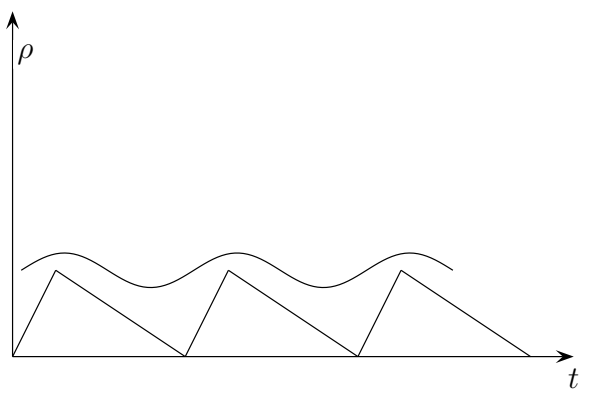

Figure 4: The sequential passing of solitons (sharp peaks) can be approximated by a sinusoidal wave form.

\section{Conclusions}

If the validity of our model is proven by experimental tests such as the pulsar timing experiments (Fig.3), the detection of time jitter (Fig.4), or the detection by an observatory such as LIGO or VIRGO of the passage of a shell front by a dedicated type of data analysis, the implications for cosmology are great. The basis of our model is that the source of dark energy is the creation of gravitational waves by the interaction of surfaces at critical density, e.g. the Planck surfaces surrounding black holes, with a strong-gravity brane located a few Planck lengths from our weak-gravity brane. Our model is consistent with the big-bang theory after the first Planck time. However, in our model the universe starts from a Lemaitre-like 'atom' or 'seed' [5].

Our model also has implications for quantum gravity theory. Experimental evidence for the validity of 
our model would imply the existence of a strong-gravity brane and extra dimensions as well as a smallest distance and impenetrable Planck surfaces rather than horizons.

Although our model describes several observed cosmological phenomena, it is largely heuristic. We are currently working on an exact solution of the fivedimensional space-time metric and a more formal mathematical description of the stimulated emission amplitude for the creation of the solitons.

\section{Acknowledgement}

Discussions with Lou Clavelli greatly contributed to the development of the paper; discussions with Laurenţiu Caramete (Bucharest, Romania), Roberto Casadio (Bologna, Italy), Marco Cavaglia (Oxford, MS), Laszlo Gergely (Szeged, Hungary), Shaoqi Hou (Tuscaloosa, AL), Pankaj Joshi (Mumbai, India), Gopal-Krishna (Pune, India), Octavian Micu (Bucharest, Romania), Piero Nicolini (Frankfurt, Germany), Norma Sanchez (Paris, France), Joe Silk (Oxford, United Kingdom), Allen Stern (Tuscaloosa, AL), Dijan Stoikovic (Buffalo, NY), and Hector de Vega (Paris, France) are gratefully acknowledged. Helpful comments on earlier versions of the manuscript were received from R. Casadio, M. Cavaglia, L. Gergely, A. Graham, P. Joshi, O. Micu, P. Nicolini, and D. Stoikovic.

This research was supported in part by the DOE under grant DE-FG02-10ER41714.

\section{References}

[1] Appenzeller, I., \& Fricke, K., A\&A 18, 10 (1972a); Appenzeller, I., \& Fricke, K., A $\& A$ 21, 285 (1972b)

[2] Bekenstein, J.D., Phys. Rev. D 7, 2333 (1973) doi:10.1103/PhysRevD .7.2333

[3] Biermann, L., Schlüter, A., Phys. Rev. 82, 863 (1951) doi:10.1103/PhysRev.82.863

[4] Biermann, P.L., Kusenko, A., Phys. Rev. Lett 96, $091301 \quad$ (2006) doi:10.1103/PhysRevLett.96.091301

[5] Biermann, P.L., \& Harms, B.C., eprint arXiv:1205.4016 (2012); Biermann, P.L., \& Harms, B.C., in Proc. at the 13th Marcel Grossmann Meeting, July 2012, eprint arXiv:1302.0040 (2013)

[6] Biermann P.L., et al., Mon. Not. Roy. Astron. Soc. 441, 1147 (2014) doi:10.1093/mnras/stu541

[7] Caramete, L.I., \& Biermann, P.L., A $\& A$ 521, id.A55 (2010)
[8] Eckart, A., et al., in THE EVOLUTION OF STARBURSTS: The 331st Wilhelm and Else Heraeus Seminar. AIP Conf. Proc. 783, 17 (2005)

[9] Frieman, J.A., Turner, M.S., \& Huterer, D., ARA\&A 46, 385 (2008) doi:10.1146/annurev . astro.46.060407.145243

[10] Greene, J.E., Barth, A.J., \& Ho, L.C., New Astro. Rev. 50, 739 (2006) doi:10.1016/j.newar.2006.06.080

[11] Harms, B.C., \& Leblanc, Y., Phys. Rev. D 46, 2334 (1992); Harms, B.C., \& Leblanc, Y., Phys. Rev. D47, 2438 (1993)

[12] LIGO and Virgo collaborations, Nature 460, 990 (2009) doi:10.1038/nature08278

[13] Munyaneza, F., \& Biermann, P.L., A\&A 436, 805 (2005); Munyaneza, F., \& Biermann, P.L., Astron. \& Astroph. Letters 458, L9 (2006)

[14] Perlmutter, S., et al., ApJ 517, 565 (1999)

[15] Planck Collaboration; Ade, P.A.R., et al., eprint arXiv:1303.5078 (2013)

[16] Predehl, K., et al., Science 336, 441 (2012)

[17] Randall, L., Sundrum, R., Phys. Rev. Lett 83, 3370 (1999a); Randall, L., Sundrum, R., Phys. Rev. Lett 83, 4690 (1999b)

[18] Riess, A.G., et al. AJ 116, 1009 (1998)

[19] Sanders, R.H., ApJ 162, 791 (1970)

[20] Schmidt, B.P., et al., ApJ 507, 46 (1998)

[21] Spitzer, L., Jr., ApJL 158, L139 (1969) doi:10.1086/180451

[22] Dimopoulos, S, Raby, S.A., Wilczek, F. Phys. Today Oct., p. 25 (1991)

\section{DISCUSSION}

MOSHE ELITZUR: Are there predictions for the effect of your model on the imprint of fluctuations on CMB and baryon acoustic oscillations?

BENJAMIN HARMS : Dark energy in our mode is due to the merging of black holes at $\mathrm{a} \simeq 50$, so well after the formation of the CMB. There may be some effect on the propagation of the CMB photons, but we have not yet worked out the exact nature of this effect. 
JIM BEALL: Can you comment on the effect these 'seed' black holes have on galaxy formation?

BENJAMIN HARMS: The main difference between our model and the 'Big Bang' theory is that our model allows for large assemblies of stars which have never merged and do not have an AGN, which have apparently been observed. 